\title{
Analytical solutions of time-fractional advection problem
}

\author{
Huimin Wang \\ Department of Mathematics, Huanghuai University, Zhumadian, Henan, 463000, China \\ 13603802316@163.com
}

Abstract: we use variational iteration method (VIM) to solve some nonlinear time-fractional advection problem. Compared to the other method, the VIM is direct and straightforward.

Keyword: time-fractional advection problem; variational iteration method; Caputo fractional derivative.

\section{Introduction}

The fractional differential equations, as generalizations of classical differential equations, are increasingly used to model problems in basic sciences and engineering. In general, there exists no method that yields an exact solution for fractional order differential equations. Only approximate solutions can be obtained. Several method have been used to solve fractional differential equations, such as Adomain decomposition method [1], homotopy perturbation method [2], homotopy analysis method [3], and variational iteration method (VIM) [4].

The VIM, which is a modified Lagrange's multiplier method [5], has been shown to solve easily, accurately and effectively a large class of linear and nonlinear fractional differential equations [1-8]. The main feature of the VIM is that the solution of a nonlinear mathematical problem with linearization assumption is used as initial approximation. Then a more highly precise approximation can be obtained. This approximation converges rapidly to an accurate solution of the fractional differential equations.

The purpose of this paper is to obtain approximate analytical solutions of the following time-fractional advection problem[10]:

$$
D_{t}^{\alpha} u(x, t)+u(x, t) u_{x}(x, t)=f(x, t)
$$

where $D^{\alpha}$ is the Caputo derivative of order $\alpha$, and $0<\alpha \leq 1$. The problem (1) arises in the description of various physical processes. Such solutions are important because numerical solutions may not identify the scientific problem under investigation.

\section{Variational Iteration Method}

To illustrate the basic concepts of the variational iteration method, we consider the following nonlinear differential equation:

$$
L u+N u=f(x, t)
$$

Where $L$ and $N$ are linear and nonlinear operators, respectively, and $f(x, t)$ is the source inhomogeneous term. According to the VIM[4-6], we can construct a correction functional as:

$$
u_{n+1}(t)=u_{n}(x, t)+\int_{0}^{t} \lambda\left(L u_{n}(x, \xi)+N \tilde{u}_{n}(x, \xi)-f(x, \xi)\right) d \xi
$$

where $\lambda$ is a general Lagrangian multiplier[5], which can be identified optimally by the variational theory, the subscript $n$ denotes the nth-order approximation, $\tilde{u}_{n}$ is considered as a restricted variation, i.e., $\delta \tilde{u}_{n}=0$. Consequently, the 
solution

$$
u=\lim _{n \rightarrow \infty} u_{n}
$$

\section{The solution of advection problem}

We first consider the homogeneous advection problem[10]:

$$
\begin{aligned}
& D_{t}^{\alpha} u+u u_{x}=0, \\
& u(x, 0)=-x .
\end{aligned}
$$

According to the VIM, we can get the following iteration formula:

$$
\mathrm{u}_{n+1}(x, t)=u_{n}(x, t)-\int_{0}^{t}\left[D_{\xi}^{\alpha} u_{n}(x, \xi)+u_{n}(x, \xi) u_{x}(x, \xi)\right] d \xi .
$$

Identifying the zeroth component $u_{0}=-x$, we get

$$
\begin{aligned}
& u_{0}=-x, \\
& u_{1}=-x-x t \\
& u_{2}=-x-2 x t+\frac{1}{\Gamma(3-\alpha)} x t^{2-\alpha}-x t^{2}-\frac{1}{3} x t^{3}, \\
& u_{3}=-x-2 x t+\frac{x}{\Gamma(4-2 \alpha)} t^{3-2 \alpha}-x t^{2}-x t^{3}-\frac{1}{6} x t^{4}+\frac{2}{\Gamma(3-\alpha)} \cdot \frac{1}{5-\alpha} t^{5-\alpha},
\end{aligned}
$$

and so on.

Remark1. when $\alpha=1$, from the above approximations, we have

$$
\begin{gathered}
u_{0}=-x, \\
u_{1}=-x-x t, \\
u_{2}=-x-x t-x t^{2}+\text { small terms }, \\
u_{3}=-x-x t-x t^{2}-x t^{3}+\text { small terms, }
\end{gathered}
$$

and so on.

Which gives 


$$
u(x, t)=-x\left(1+t+t^{2}+\cdots\right)=\frac{x}{t-1} .
$$

The function (5) is a exact solution of the following class Cauchy problem[10]:

$$
\left\{\begin{array}{l}
u_{t}+u u_{x}=0 \\
u(x, 0)=-x
\end{array} .\right.
$$

Next, we consider the nonhomogeneous advection problem.

$$
\left\{\begin{array}{c}
D_{\alpha} u+u u_{x}=2 t+x t^{2}+\frac{\partial^{2+\alpha}}{\Gamma(2+\alpha)}+\frac{x}{\Gamma(2-\alpha)} t^{1-\alpha} \\
u(x, 0)=0
\end{array} .\right.
$$

Proceeding as before, we have

$$
\begin{aligned}
u_{n+1}(x, t)= & \frac{2}{\Gamma(2+\alpha)} t^{1+\alpha}+\frac{2 x}{\Gamma(3+\alpha)} t^{2+\alpha}+x t+\frac{\alpha 2+\alpha)}{\Gamma(3+2 \alpha)} t^{2+2 \alpha} \\
& +\frac{1}{\Gamma(\alpha)} \int_{0}^{t}(t-\xi)^{\alpha-1}\left(-u_{n}(x, \xi) u_{n x}(x, \xi)\right) d \xi
\end{aligned}
$$

Taking $u_{0}=0$, by(7), we get

$$
\begin{aligned}
& u_{1}=\frac{2}{\Gamma(2+\alpha)} t^{1+\alpha}+x t+\text { small terms } \\
& u_{2}=\frac{2}{\Gamma(2+\alpha)} t^{1+\alpha}+x t+\text { small terms }
\end{aligned}
$$

and so on.

Thus we get

$$
u=\lim _{n \rightarrow \infty} u_{n}=\frac{2}{\Gamma(2+\alpha)} t^{1+\alpha}+x t
$$

Which gives the exact solution of problem (6).

Remark 2. when $\alpha=1$, from the above results, we get

$$
\begin{gathered}
u_{0}=0, \\
u_{1}=t^{2}+x t+\text { small terms, }
\end{gathered}
$$




$$
u_{2}=t^{2}+x t+\text { small terms }
$$

and so on.

Thus the function

$$
u(x, t)=t^{2}+x t
$$

Is the exact solution of the following class advection Cauchy problem:

$$
\left\{\begin{array}{c}
u_{t}+u u_{x}=2+x+t^{3}+x t^{2} \\
u(x, 0)=0
\end{array}\right.
$$

\section{Conclusion}

Variational iteration method gives several successive approximations through using the iteration of the correction functional, and more importantly, it reduces the volume of calculations via not requiring Adomain polynomials[9], thus VIM is direct and straightforward.

\section{References}

1. Adomian G. A review of the decomposition method in applied mathematics[J]. Journal of mathematical analysis and applications, 1988, 135(2): 501-544.

2. He $\mathrm{J} \mathrm{H}$. Homotopy perturbation method: a new nonlinear analytical technique[J]. Applied Mathematics and computation, 2003, 135(1): 73-79.

3. Liao S. Beyond perturbation: introduction to the homotopy analysis method[M]. CRC press, 2003.

4. He $\mathrm{J} \mathrm{H}$. Variational iteration method-a kind of non-linear analytical technique: some examples[J]. International journal of non-linear mechanics, 1999, 34(4): 699-708.

5. Nash S G. Calculus of Variations[J]. Progress in Nonlinear Differential Equations \& Their Applications, 2008, 12(3):141-183.

6. Momani S, Abuasad S. Application of He's variational iteration method to Helmholtz equation[J]. Chaos Solitons \& Fractals, 2006, 27(5):1119-1123.

7. Odibat Z, Momani S. The variational iteration method: An efficient scheme for handling fractional partial differential equations in fluid mechanics[J]. Computers \& Mathematics with Applications, 2009, 58(11-12):2199-2208.

8. Wazwaz A M. A study on linear and nonlinear Schrodinger equations by the variational iteration method[J]. Chaos Solitons \& Fractals, 2008, 37(4):1136-1142.

9. Elsaid A. Fractional differential transform method combined with the Adomian polynomials[J]. Applied Mathematics and Computation, 2012, 218(12): 6899-6911.

10. Wazwaz A M. A new approach to the nonlinear advection problem: An application of the decomposition method[J]. Applied Mathematics \& Computation, 1995, 72(2-3):175-181. 